\title{
Bioinformatics Interpretation of Exome Sequencing: Blood Cancer
}

\author{
Jiwoong Kim ${ }^{1}$, Yun-Gyeong Lee ${ }^{1,2}$, Namshin Kim ${ }^{1,2}$ \\ ${ }^{1}$ Korean Bioinformation Center, Korea Research Institute of Bioscience and Biotechnology, Daejeon 305-806, Korea, \\ ${ }^{2}$ Department of Bioinformatics, University of Science and Technology, Daejeon 305-806, Korea
}

http://dx.doi.org/10.5808/GI.2013.11.1.24

Genomics Inform 2013;11(1):24-33

The funding acknowledgment in this article was partially omitted as published. Additional acknowledgment is as follows:

This work was supported by a National Research Foundation of Korea (NRF) grant funded by the Korea government (MEST) (nos. 20110030770 and 20120009215), a grant from the Next-Generation BioGreen 21 Program (nos. PJ008019 and PJ008068), Rural Development Administration, a grant from KRIBB Research Initiative Program, Republic of Korea, and a grant of the National Project for Personalized Genomic Medicine, Ministry for Health \& Welfare, Republic of Korea (A111218). 